\title{
Chaos in the Kepler System
}

\author{
C. Chicone* , B. Mashhoon ${ }^{\dagger}$ and D. G. Retzloff ${ }^{\dagger}$
}

\begin{abstract}
The long-term dynamical evolution of a Keplerian binary orbit due to the emission and absorption of gravitational radiation is investigated. This work extends our previous results on transient chaos in the planar case to the three dimensional Kepler system. Specifically, we consider the nonlinear evolution of the relative orbit due to gravitational radiation damping as well as external gravitational radiation that is obliquely incident on the initial orbital plane. The variation of orbital inclination, especially during resonance capture, turns out to be very sensitive to the initial conditions. Moreover, we discuss the novel phenomenon of chaotic transition.
\end{abstract}

\section{INTRODUCTION}

In a recent investigation of the radiative perturbations of the planar Kepler problem [1], we have found evidence for transient chaos. Specifically, we have considered in our previous work [1, 2] an isolated binary system consisting of masses $m_{1}$ and $m_{2}$ whose internal structures have been ignored, i.e. they have been treated essentially as Newtonian point masses with positions $\mathbf{x}_{1}$ and $\mathbf{x}_{2}$ in an inertial frame. The relative motion is damped since orbital energy and angular momentum leave the system due to the emission of gravitational radiation. Moreover, the binary system is perturbed by an external normally incident plane gravitational wave. All radiative perturbations of the binary system have been treated in the quadrupole approximation in our work [1]; therefore, it follows that the center-ofmass motion is unaffected by the radiative effects. The well-known connection between the Kepler system and the harmonic oscillator implies that the system under consideration is analogous to a certain damped oscillator with external periodic forcing. In fact, it is expected that replacing the emission and absorption of gravitational radiation by other similar damping and forcing mechanisms, respectively, might lead to similar phenomena. For instance, the influence of the external radiation could be replaced by the tidal perturbations of a distant third mass.

The transient chaos in the dynamical evolution of the relative orbit under radiative perturbations appears to be associated with the phenomenon of capture into resonance as explained in detail in our present work. Moreover, we point out in this paper an interesting aspect of transient chaos, namely, the phenomenon of chaotic transition. The slow evolution of the binary orbit while locked in resonance can be explained using the second order averaged dynamics [2] that is described here for the three dimensional Kepler system.

It is important to emphasize the idealized nature of the model under investigation here [1 1 - 4 . We consider the simplest Keplerian model in which the effects of emission and absorption of gravitational radiation are taken into account. The radiative damping is of particular interest in view of the timing observations of the Hulse-Taylor relativistic binary pulsar PSR B1913+16: the data can be interpreted in terms of energy loss due to the emission of gravitational radiation in agreement with general relativity [5.6]. For recent discussions of the measurable relativistic effects in binary systems, we refer to the investigations of Kopeikin and his collaborators [7.

It is estimated that about half of all stars are members of binary or multiple systems; therefore, it is possible that the general phenomena we have encountered in our theoretical investigations have actually occurred in nature. In particular, our results [1 4 should be relevant for the behavior of relativistic binary pulsars; indeed, a number of such systems have been discovered since the existence of the original binary pulsar PSR B1913+16 was first recognized [5, 8]. This possibility provides the impetus to analyze the Kepler system under more general conditions, especially in connection with the nature of its chaotic behavior. To this end, we consider in this paper the radiative perturbations of the three dimensional Kepler system. As work continues on relativistic binary systems-following the Taylor-Hulse work on the original binary pulsar - and more are discovered and studied, it may be that the theoretical results presented in our work could be useful in the elucidation of observed phenomena in such interesting astronomical systems.

\footnotetext{
* Department of Mathematics, University of Missouri, Columbia, MO 65211. Supported by the NSF grant DMS-9531811 and the University of Missouri Research Board.

${ }^{\dagger}$ Department of Physics and Astronomy, University of Missouri, Columbia, MO 65211.

${ }^{\ddagger}$ Department of Chemical Engineering, University of Missouri, Columbia, MO 65211.
} 


\section{DYNAMICAL EQUATIONS}

The simplest equation of relative motion for the Kepler system including radiative perturbations is given by

$$
\frac{d^{2} x^{i}}{d t^{2}}+k \frac{x^{i}}{|\mathbf{x}|^{3}}+\mathcal{R}^{i}=-\epsilon \mathcal{K}_{i j}(t) x^{j}
$$

where $\mathbf{x}:=\mathbf{x}_{1}-\mathbf{x}_{2}$ is the vector describing relative motion, $k:=G_{0} M, M:=m_{1}+m_{2}$, the quantity $-\mathcal{R}$ is the relative acceleration caused by gravitational radiation reaction, and $\epsilon \mathcal{K}$ is the tidal matrix of the external wave evaluated at the center of mass of the binary system that we take to be the origin of spatial coordinates in the background inertial frame. Thus, in this frame the motion of $m_{1}$ and $m_{2}$ can be described by $\mathbf{x}_{1}(t)=\left(m_{2} / M\right) \mathbf{x}(t)$ and $\mathbf{x}_{2}(t)=-\left(m_{1} / M\right) \mathbf{x}(t)$, respectively. Various properties of the system (11) have been discussed in detail in our recent investigations of the planar Kepler problem [1] 4 ; therefore, we concentrate in this paper on the long term deviations of the system (1) from planar motion.

In the quadrupole approximation, the standard expression for radiation damping may be reduced-for the Kepler system under consideration - to

$$
\mathcal{R}=\frac{4 G_{0}^{2} m_{1} m_{2}}{5 c^{5}|\mathbf{x}|^{3}}[\psi \mathbf{v}-\chi(\hat{\mathbf{x}} \cdot \mathbf{v}) \hat{\mathbf{x}}]
$$

where $\mathbf{v}=d \mathbf{x} / d t$ is the relative velocity, $\hat{\mathbf{x}}=\mathbf{x} /|\mathbf{x}|$, and

$$
\psi=12 v^{2}-30(\hat{\mathbf{x}} \cdot \mathbf{v})^{2}-\frac{4 k}{|\mathbf{x}|}, \quad \chi=36 v^{2}-50(\hat{\mathbf{x}} \cdot \mathbf{v})^{2}+\frac{4 k}{3|\mathbf{x}|} .
$$

Moreover, the tidal matrix for a plane monochromatic wave of frequency $\Omega$ propagating in the $\left(x^{1}, x^{3}\right)$-plane along a direction that makes an angle $\Theta, 0 \leq \Theta \leq \pi$, with respect to the $x^{3}$-axis is given by (cf. appendix A)

$$
\begin{aligned}
& \mathcal{K}_{11}=\alpha \Omega^{2} \cos ^{2} \Theta \cos \Omega t \\
& \mathcal{K}_{12}=\beta \Omega^{2} \cos \Theta \cos \left(\Omega t+\phi_{0}\right), \\
& \mathcal{K}_{13}=-\alpha \Omega^{2} \cos \Theta \sin \Theta \cos \Omega t \\
& \mathcal{K}_{22}=-\alpha \Omega^{2} \cos \Omega t \\
& \mathcal{K}_{23}=-\beta \Omega^{2} \sin \Theta \cos \left(\Omega t+\phi_{0}\right),
\end{aligned}
$$

and the other components of the tidal matrix are determined by the fact that $\left(\mathcal{K}_{i j}\right)$ is symmetric and traceless. Here $\alpha$ and $\beta$ are amplitudes of the two independent linear polarization states of the incident radiation and $\phi_{0}$ is the constant phase difference between them. The background inertial frame is fixed by the form of the tidal matrix (4) and the fact that the initial unperturbed orbit is assumed to be in the $\left(x^{1}, x^{2}\right)$-plane. The strength of the external perturbation is given by $\epsilon, 0<\epsilon<<1$, so that $\alpha$ and $\beta$ are of the order of unity; at present, it is expected that $\epsilon \sim 10^{-20}$ though gravitational waves have not yet been detected in the laboratory.

It must be emphasized that the simple form of the external perturbation assumed here is meant to represent the dominant component of a wave packet composed mostly of wavelengths much larger than the size of the Keplerian system. In the absence of the external perturbation, an orbit in the $\left(x^{1}, x^{2}\right)$-plane would remain confined to this plane under radiative damping. If the external wave is turned on at a certain instant, it is expected that the equation of motion (1) would represent the steady-state situation after transients have died away.

It is interesting to subject the equation of motion to certain scale transformations that would render it dimensionless. To this end, let $\mathbf{x}=R_{0} \tilde{\mathbf{x}}$ and $t=T_{0} \tilde{t}$ such that $R_{0}$ and $T_{0}$ are constants and a tilde denotes a dimensionless quantity. In this way the equation of motion keeps its form except that $k$ must be replaced by $\tilde{k}$,

$$
\tilde{k}=k \frac{T_{0}^{2}}{R_{0}^{3}},
$$

$\Omega=\tilde{\Omega} / T_{0}$ and the strength of the gravitational radiation reaction is given by the dimensionless quantity $\delta$,

$$
\delta=\frac{4 G_{0}^{2} m_{1} m_{2}}{5 c^{5} T_{0} R_{0}}
$$

In the rest of this paper, we fix the relationship between $R_{0}$ and $T_{0}$ by $k T_{0}^{2}=R_{0}^{3}$, so that $\tilde{k}=1$. Thus if $R_{0}$ is taken to be the semimajor axis of the initial Keplerian orbit, then its period is given by $2 \pi T_{0}$. We adopt this convention 
regarding the initial orbit throughout this work. It follows that $\delta<(20 \sqrt{2})^{-1}$ in all physically significant cases; in fact, $\delta \sim 10^{-15}$ for the binary pulsar PSR B1913 +16 [5, 6]. Henceforth we deal with dimensionless equations of motion in which all tildes are dropped.

It is appropriate to introduce cylindrical coordinates $(\rho, \theta, z)$ such that $x^{1}=\rho \cos \theta, x^{2}=\rho \sin \theta$, and $x^{3}=z$. The equations of motion can then be expressed in the form

$$
\begin{aligned}
& \frac{d \rho}{d t}=p_{\rho}, \quad \frac{d \theta}{d t}=\frac{p_{\theta}}{\rho^{2}}, \quad \frac{d z}{d t}=p_{z} \\
& \frac{d p_{\rho}}{d t}=-\frac{\rho}{\left(\rho^{2}+z^{2}\right)^{3 / 2}}+\frac{p_{\theta}^{2}}{\rho^{3}}-\mathcal{R}^{\rho} \\
& \quad-\epsilon \rho\left[\frac{1}{2}\left(\mathcal{K}_{11}+\mathcal{K}_{22}\right)+\frac{1}{2}\left(\mathcal{K}_{11}-\mathcal{K}_{22}\right) \cos 2 \theta+\mathcal{K}_{12} \sin 2 \theta\right] \\
& \quad-\epsilon z\left(\mathcal{K}_{13} \cos \theta+\mathcal{K}_{23} \sin \theta\right), \\
& \frac{d p_{\theta}}{d t}=-\mathcal{R}^{\theta}-\epsilon \rho^{2}\left[\mathcal{K}_{12} \cos 2 \theta-\frac{1}{2}\left(\mathcal{K}_{11}-\mathcal{K}_{22}\right) \sin 2 \theta\right] \\
& \quad-\epsilon \rho z\left(-\mathcal{K}_{13} \sin \theta+\mathcal{K}_{23} \cos \theta\right), \\
& \frac{d p_{z}}{d t}=- \frac{z}{\left(\rho^{2}+z^{2}\right)^{3 / 2}}-\mathcal{R}^{z}-\epsilon \rho\left(\mathcal{K}_{13} \cos \theta+\mathcal{K}_{23} \sin \theta\right)-\epsilon z \mathcal{K}_{33},
\end{aligned}
$$

where we use equation (ब) for the external perturbation and

$$
\mathcal{K}_{33}=-\left(\mathcal{K}_{11}+\mathcal{K}_{22}\right)=\alpha \Omega^{2} \sin ^{2} \Theta \cos \Omega t .
$$

In equation (7), the radiation reaction terms are given by

$$
\begin{aligned}
& \mathcal{R}^{\rho}=\frac{\delta}{\left(\rho^{2}+z^{2}\right)^{3 / 2}}\left[\psi p_{\rho}-\chi \frac{\rho\left(\rho p_{\rho}+z p_{z}\right)}{\rho^{2}+z^{2}}\right], \\
& \mathcal{R}^{\theta}=\frac{\delta \psi p_{\theta}}{\left(\rho^{2}+z^{2}\right)^{3 / 2}}, \\
& \mathcal{R}^{z}=\frac{\delta}{\left(\rho^{2}+z^{2}\right)^{3 / 2}}\left[\psi p_{z}-\chi \frac{z\left(\rho p_{\rho}+z p_{z}\right)}{\rho^{2}+z^{2}}\right],
\end{aligned}
$$

where $\psi$ and $\chi$ are given by equation (3) and can be expressed as

$$
\begin{aligned}
& \psi=12\left(p_{\rho}^{2}+\frac{p_{\theta}^{2}}{\rho^{2}}+p_{z}^{2}\right)-30 \frac{\left(\rho p_{\rho}+z p_{z}\right)^{2}}{\rho^{2}+z^{2}}-\frac{4}{\left(\rho^{2}+z^{2}\right)^{1 / 2}}, \\
& \chi=36\left(p_{\rho}^{2}+\frac{p_{\theta}^{2}}{\rho^{2}}+p_{z}^{2}\right)-50 \frac{\left(\rho p_{\rho}+z p_{z}\right)^{2}}{\rho^{2}+z^{2}}+\frac{4}{3\left(\rho^{2}+z^{2}\right)^{1 / 2}} .
\end{aligned}
$$

It now remains to integrate these equations with appropriate boundary conditions.

\section{NUMERICAL EXPERIMENTS}

The equations of motion (7) have been so formulated that for $\Theta=0$, we recover the results of our recent work [1,2. We wish to investigate how our previous results vary with $\Theta$ as the direction of incidence of the external radiation deviates from the normal. It is clear that our previous theoretical results, such as regarding the persistence of periodic orbits, would still hold for sufficiently small $\Theta, 0<\Theta \ll 1$; however, the analysis for arbitrary $\Theta$ would be cumbersome and we therefore resort to numerical experiments. To express our numerical results in a form that could be easily compared with previous results [1.2], we note that the energy $E$, total angular momentum $G$ and the $z$-component of

the angular momentum $H$ for the osculating ellipse in our system can be expressed in terms of cylindrical coordinates as

$$
\begin{aligned}
E & =\frac{1}{2}\left(p_{\rho}^{2}+\frac{p_{\theta}^{2}}{\rho^{2}}+p_{z}^{2}\right)-\frac{1}{\left(\rho^{2}+z^{2}\right)^{1 / 2}}, \\
G & =\left[\left(\rho p_{z}-z p_{\rho}\right)^{2}+\left(1+\frac{z^{2}}{\rho^{2}}\right) p_{\theta}^{2}\right]^{1 / 2}, \\
H & =p_{\theta},
\end{aligned}
$$


respectively. The corresponding Delaunay elements are the action variables $(L, G, H)$, where $L=(-2 E)^{-1 / 2}$. The osculating ellipse is depicted in figure 1 .

Some typical results of our numerical experiments are presented in figures 2 6 . Figure 2 explores the phenomenon associated with capture into resonance; here the osculating orbit has eccentricity $e, G=L\left(1-e^{2}\right)^{1 / 2}$, and $H=G \cos i$, where $i$ denotes the orbital inclination. The angle of incidence is $\Theta=\pi / 10$. Figures 36 explore the phenomena associated with transient chaos for $\Theta=\pi / 6$.

To arrive at these representative results, we begin our numerical work with a resonant orbit given at some initial instant of time by

$$
\left(p_{\rho}, p_{\theta}, p_{z}, \rho, \theta, z\right)=\left(e_{0}, 1,0,1,0,0\right),
$$

which corresponds to an osculating ellipse in the $\left(x^{1}, x^{2}\right)$-plane with eccentricity $e_{0}$, unit orbital angular momentum about the $z$-axis, semimajor axis $\left(1-e_{0}^{2}\right)^{-1}$ and Keplerian frequency $\left(1-e_{0}^{2}\right)^{3 / 2}$. In figure 1 , for instance, this initial orbit would have $h=0, g=-\pi / 2$ and $\hat{v}=\pi / 2$. We then assume an external wave that is a coherent superposition of the two independent linear polarization states with equal amplitudes $(\alpha=\beta)$ and zero phase shift between them $\left(\phi_{0}=0\right)$. The wave is incident at an angle $\Theta$ that we vary in our experiments. We choose the wave frequency $\Omega=m \omega$ to correspond to the $(m: 1)$ resonance. Integrating forward and backward from this initial system, we explore the region around the resonance configuration. Starting from a state before resonance capture, we then integrate forward until the integration routine gives up due to the rapid collapse of the binary system. To compare our results with the planar case $\Theta=0$, we choose the two planar configurations with initial $e_{0}=1 / 2$ that we studied in a previous paper [1]. In the first system, i.e. figure 1 of [1], we used $\epsilon=10^{-4}, \delta=10^{-7}, \alpha=\beta=2, \phi_{0}=0$ and $\Omega=L_{0}^{-3}$ with $L_{0}=\left(1-e_{0}^{2}\right)^{-1 / 2}$. This $(1: 1)$ resonance is studied for $\Theta=\pi / 10$ in figure 2 of the present paper. In the second system, i.e. figure 2 of [1], we used $\epsilon=10^{-3}, \delta=10^{-8}, \alpha=\beta=2, \phi_{0}=0$ and $\Omega=2 L_{0}^{-3}$ with $L_{0}=\left(1-e_{0}^{2}\right)^{-1 / 2}$. This $(2: 1)$ resonance is studied for $\Theta=\pi / 6$ in figures $3 / 6$ of the present paper; in these figures, every tenth iterate of the $2 \pi / \Omega$ stroboscopic Poincaré map is plotted. This second case exhibits transient chaos and to be reasonably certain of the various features of this chaotic regime, we have used two different standard routines for stiff integration of (77) using double precision arithmetic. The results of the integration of this configuration are depicted in figures 3 and 4 . These are to be compared with figures 5 and 6 , respectively, which depict the results of integration of essentially the same system albeit with a different starting point. It is important to note that the orbital inclination is particularly sensitive to transient chaos as is clear from the behavior of $\cos i$ in figures 1 and 6 . Moreover, as the binary system collapses, we expect that eventually the binary orbit would tend to a circle $(e \rightarrow 0)$. In figures 36 the endpoint of integration exhibits a sharp drop in eccentricity as would be theoretically expected. Note that this behaviour of eccentricity coincides with the sharp bend in $L(t)$ near $L=1$ corresponding to chaotic transition as described in section $\mathrm{V}$.

In the following sections, we present a theoretical explanation of some of the main features of the behavior of the system over a long period of time. For this purpose, essential use is made of the dynamical equations in terms of Delaunay elements (cf. appendix B).

\section{AVERAGING}

The long-term behavior of the dynamical system under investigation is best revealed after we average the system over the Keplerian frequency $\omega=L^{-3}$. In fact, the Delaunay equations (cf. appendix B) show clearly that the three dimensional Kepler system has only one intrinsic frequency, namely, $\omega$. Let us write the equations of motion in the form

$$
\begin{aligned}
\frac{d L}{d t} & =-\epsilon \frac{\partial \mathcal{H}^{*}}{\partial \ell}+\epsilon \Delta f_{L}, \\
\frac{d G}{d t} & =-\epsilon \frac{\partial \mathcal{H}^{*}}{\partial g}+\epsilon \Delta f_{G}, \\
\frac{d H}{d t} & =-\epsilon \frac{\partial \mathcal{H}^{*}}{\partial h}+\epsilon \Delta f_{H}, \\
\frac{d \ell}{d t} & =\frac{1}{L^{3}}+\epsilon \frac{\partial \mathcal{H}^{*}}{\partial L}+\epsilon \Delta f_{\ell}, \\
\frac{d g}{d t} & =\epsilon \frac{\partial \mathcal{H}^{*}}{\partial G}+\epsilon \Delta f_{g}, \\
\frac{d h}{d t} & =\epsilon \frac{\partial \mathcal{H}^{*}}{\partial H}+\epsilon \Delta f_{h},
\end{aligned}
$$




$$
\frac{d \tau}{d t}=\Omega
$$

where $\mathcal{H}^{*}$ is the Hamiltonian associated with the external perturbation, $\Delta=\delta / \epsilon$ and $f_{D}, D \in\{L, G, H, \ell, g, h\}$, denotes the gravitational radiation damping. Here $\tau=\Omega t$ is a new angular variable.

In the absence of the external perturbation, we have shown [1] that the system maintains its orbital plane - i.e., the inclination of the orbit remains fixed - but loses energy and angular momentum so that the orbit decays monotonically and eventually collapses. During this process the orbit tends to a circle, i.e. the eccentricity $e$ decreases. With the external radiation present, as in (11), we have a dynamical system with two frequencies $\omega$ and $\Omega$ and the possibility of resonance needs to be taken into account. This would occur if relatively prime integers $m$ and $n$ exist such that $m \omega=n \Omega$; the $(m: n)$ resonance manifold is then given by the set

$$
\left\{(L, G, H, \ell, g, h, \tau): m \frac{1}{L^{3}}=n \Omega\right\} .
$$

Let us first assume that the dynamical system (14) is off resonance; then, averaging (14) over a period of the external radiation results in a system that is simply damped. That is, the terms involving $\mathcal{H}^{*}$ in (14) simply drop out; therefore, the system tends to a circular orbit as it decays on the average while maintaining the inclination of the orbital plane (cf. appendix B). This explains figure 2 before and after resonance capture; that is, in these regimes $e$ decreases monotonically on the average while $\cos i$ is essentially constant. If the resonance condition is satisfied, then the system either passes through the resonance without being captured or is captured into the resonance. In the former case, the system behaves on the average in the same way that it would off resonance. In the latter case, however, the resonance condition fixes the semimajor axis of the osculating ellipse on the average. Thus the external wave deposits energy into the system so as to balance radiation damping on the average and hence $L$ remains fixed on the average while the exchange of angular momentum takes place. The resulting change in $G$ and $H$ affects the shape as well as the configuration of the orbit; in fact, the eccentricity and the inclination of the orbit vary while its semimajor axis oscillates about the value fixed by the resonance. This is clearly indicated in figure 2, where the inclination and the eccentricity of the orbit both decrease as a result of passage through resonance. The rate of damping depends on the shape of the orbit and this fact accounts for the difference in the slope of $L(t)$ before and after capture into resonance. During resonance capture, we let $L=L_{0}+\epsilon^{1 / 2} \mathcal{D}$ and $\ell=\phi+n \Omega t / m$, where $L_{0}^{-3}=n \Omega / m$ and $\mathcal{D}$ and $\phi$ are the new canonical variables associated with resonance. The behavior of the dynamical system while trapped in resonance can be studied using the method of partial averaging (cf. appendix C). It turns out that for the radiative perturbations of the three dimensional Kepler system under consideration here resonance is possible only for $n=1$. Numerical experiments then reveal that the $(1: 1)$ resonance has a simple structure as in figure 2 . More complicated structures are expected for higher order resonances as described in our previous planar work [2]. In the present paper, we explore a $(2: 1)$ resonance in figures $3-6$. A general discussion of the $(m: 1)$ resonance for the Kepler system is beyond the scope of this work.

To explain the behavior of the system while trapped in resonance in a quantitative manner, we need to develop the first and second order partially averaged dynamics for (14) following the method adapted in our recent work on the planar Kepler system [2]. This is done in appendix C and the resulting system of second order partially averaged Delaunay equations for the $(m: 1)$ resonance are

$$
\begin{aligned}
\dot{\mathcal{D}}=- & \epsilon^{1 / 2}\left[-m \mathcal{T}_{c} \sin m \phi+m \mathcal{T}_{s} \cos m \phi+\frac{\Delta}{G^{7}}\left(8+\frac{73}{3} e^{2}+\frac{37}{12} e^{4}\right)\right] \\
& -\epsilon \mathcal{D}\left[-m \frac{\partial \mathcal{T}_{c}}{\partial L} \sin m \phi+m \frac{\partial \mathcal{T}_{s}}{\partial L} \cos m \phi+\frac{\Delta}{3 L_{0}^{3} G^{5}}\left(146+37 e^{2}\right)\right], \\
\dot{G}=-\epsilon & {\left[\frac{\partial \mathcal{T}_{c}}{\partial g} \cos m \phi+\frac{\partial \mathcal{T}_{s}}{\partial g} \sin m \phi+\frac{\Delta}{L_{0}^{3} G^{4}}\left(8+7 e^{2}\right)\right], } \\
\dot{H}=-\epsilon & {\left[\frac{\partial \mathcal{T}_{c}}{\partial h} \cos m \phi+\frac{\partial \mathcal{T}_{s}}{\partial h} \sin m \phi+\frac{\Delta}{L_{0}^{3} G^{4}}\left(8+7 e^{2}\right) \cos i\right], } \\
\dot{\phi}=-\epsilon^{1 / 2} \frac{3}{L_{0}^{4}} \mathcal{D}+\epsilon\left(\frac{6}{L_{0}^{5}} \mathcal{D}^{2}+\frac{\partial \mathcal{T}_{c}}{\partial L} \cos m \phi+\frac{\partial \mathcal{T}_{s}}{\partial L} \sin m \phi\right), & \left(\frac{\partial \mathcal{T}_{c}}{\partial G} \cos m \phi+\frac{\partial \mathcal{T}_{s}}{\partial G} \sin m \phi\right), \\
\dot{g}=\epsilon\left(\frac{\partial \mathcal{T}_{c}}{\partial H} \cos m \phi+\frac{\partial \mathcal{T}_{s}}{\partial H} \sin m \phi\right), &
\end{aligned}
$$


where $\mathcal{T}_{c}$ and $\mathcal{T}_{s}$ are complicated expressions involving $\alpha, \beta, \Omega, \phi_{0}, \Theta$ and the Delaunay elements given by (C25) in appendix C. It is important to note that the first-order averaged equations are formally the same as in [2]; that is, to order $\epsilon^{1 / 2}$ the orbital inclination is fixed and we simply have an oscillator in $\mathcal{D}$ and a pendulum with constant torque in $\phi$ as before. To second order in $\epsilon^{1 / 2}$, the damping (or antidamping) term enters the pendulum equation and this results in an antidamped (or damped) harmonic oscillator in $\mathcal{D}$ as in the case illustrated in figure 2 . These features have been explained in detail in [2]; therefore, it is more interesting to investigate the new aspects of resonance capture associated with the slow oscillation of the plane of the osculating orbit. In fact, the possibility of the variation of the inclination to second order in $\epsilon^{1 / 2}$ would result in an additional feature that is required for a complete explanation of figure 2. The pendulum in $\phi$ will couple, in the general case, to an equation for the variation in the orbital inclination. To develop this latter equation, we note that $H=G \cos i$ and hence $d(\cos i) / d t=(\dot{H} G-H \dot{G}) / G^{2}$, where $\dot{G}$ and $\dot{H}$ are given by (14). We are interested in the slow variation in the inclination of the orbit during resonance capture. The average behavior of the orbital inclination is obtained if we substitute for $\dot{G}$ and $\dot{H}$ from the second order partially averaged system (15) instead. In this way, we find

$$
\frac{d}{d t} \cos i=-\frac{\epsilon}{G}\left[\left(\frac{\partial \mathcal{T}_{c}}{\partial h}-\frac{\partial \mathcal{T}_{c}}{\partial g} \cos i\right) \cos m \phi+\left(\frac{\partial \mathcal{T}_{s}}{\partial h}-\frac{\partial \mathcal{T}_{s}}{\partial g} \cos i\right) \sin m \phi\right],
$$

for the slow variation of the orbital inclination while the system is captured in resonance. It is remarkable that the explicit damping terms proportional to $\Delta$ in (15) drop out in equation (16); indeed, in the absence of external waves there would be no resonance capture and $\cos i$ would be constant. Thus the rate of variation of $\cos i$ is directly proportional to the presence of the external perturbation as in (16). It is difficult to draw exact conclusions regarding $\cos i$ from (15) and (16); however, we intuitively expect that the orbital inclination would undergo on average simple oscillatory movements during resonance capture. These oscillations are expected to be slow, since cos $i$ varies in time over the temporal scale given by $\epsilon t$. These conclusions are generally consistent with the results of our numerical experiments.

\section{TRANSIENT CHAOS}

Numerical experiments suggest that our system exhibits chaotic transients near the "exit from resonance". In our experiments, we considered the $(2: 1)$ resonance similar to the planar case in figure 2 of [1]. Starting at the point $\left(p_{\rho}, p_{\theta}, p_{z}, \rho, \theta, z\right)=(0.5,1,0,1,0,0)$ which is near the resonance manifold, backward integration shows that the system is captured into resonance; then, after a sufficiently long forward integration, the orbit leaves the vicinity of the resonance and continues toward collapse. However, the length of time required for the system to exit the resonance is very sensitive to the integration method and to changes in the initial conditions. Also, plots of $L$ versus $t$ show that the signal appears to pass through a chaotic region after it finally exits the resonance. This strongly suggests that the system is passing through an unstable chaotic set; that is, there is transient chaos.

We expect transient chaotic effects to be present throughout the resonance capture region; however, in our experiments transient chaos appears most prominently after exit from resonance. Following the exit from the $(2: 1)$ resonance under consideration, the system collapses relatively slowly until it undergoes a certain chaotic transition and the relative orbit that emerges after this transition collapses much more rapidly. In fact, the system makes a transition from one relative orbit to a totally different relative orbit in a rather short period of time while undergoing what appears to be transient chaos. For lack of a better characterization of this unexpected phenomenon, we refer to it as "chaotic transition". As a consequence of this transformation, a "bent knee" appears in figures 3 (6 near $L=1$; indeed, the rate at which the system collapses suddenly changes at the "bend". That is, the orbital eccentricity increases very rapidly and this leads to a more rapid rate of loss of energy due to the emission of gravitational radiation - since this is proportional to $\left(1-e^{2}\right)^{-7 / 2}$ as can be seen from inspection of equation (15) of [1] - and hence the "bend." We have verified by two different numerical methods that these chaotic phenomena are stable features of the $(2: 1)$ resonance.

\section{CONCLUSION}

We consider in this paper a three dimensional Kepler system that is subject to radiative perturbations caused by radiation damping as well as an incident monochromatic gravitational wave.

A Keplerian binary system constantly loses energy to gravitational radiation according to general relativity. There is a lack of complete reciprocity between the emission and absorption of gravitational radiation, however. The absorption of gravitational radiation by the binary is not monotonic and the system sometimes gains energy from the external 
wave and sometimes deposits energy into the wave. The behavior of the system averaged over the period of the external wave is thus one of continuous collapse due to radiation damping except when it is captured into resonance. Other than at resonance, the average behavior of the system is that the orbital plane remains fixed while the system shrinks as it tends to a circle $(a \rightarrow 0, e \rightarrow 0)$. At resonance, the system on average steadily gains energy from the external wave in order to balance the steady loss of energy to radiation. The resonance condition $m \omega=n \Omega$ is necessary but not sufficient for the occurrence of this delicate balance at the $(m: n)$ resonance. In general, transient chaotic behavior is expected near a resonance. As the system evolves, it passes through and is affected by a dense set of $(m: n)$ resonances. When it is indeed captured into a resonance, we find that $n=1$.

We have not proven the existence of transient chaos in the Kepler system when radiative perturbations are taken into account. Instead, we have presented further numerical evidence in support of this hypothesis in this paper. Two different codes for the numerical integration of equation (7) have been employed and they have yielded qualitatively the same results. Our conclusions regarding transient chaos and chaotic transition are based on these numerical results for the three dimensional Kepler system.

The parameter space of the problem under consideration is very large. No significant attempt has been made at exploring this parameter space; for instance, we cannot exclude the possibility of existence of a strange attractor in our system. Instead, we have concentrated our attention on a $(1: 1)$ resonance and a $(2: 1)$ resonance in the three dimensional case that are familiar from our previous planar work [1, 2]. The (1:1) resonance has a simple structure and its general features can be explained by the second order averaged dynamics. That is, the semimajor axis undergoes antidamped oscillations about its resonance value while the orbital inclination slowly oscillates until the system leaves the resonance; on the other hand, angular momentum is transferred to the binary orbit during resonance such that the orbit after the resonance is substantially different in eccentricity from the orbit before it gets captured into resonance and hence so is the rate of collapse of the binary due to the emission of gravitational radiation.

The $(2: 1)$ resonance has a rich structure and much of our numerical analysis has been concerned with its elucidation. It appears that this structure is associated with transient chaos. Once the system leaves the resonance, the general trend towards collapse is accompanied by complex structure that we characterize as transient chaotic behavior. This is followed by a peculiar chaotic transition which may be another manifestation of transient chaos. The result is that the system collapses extremely rapidly as a result of going through this transition. Integrating backward in time from the resonance, the system grows in size. Since the radiation reaction force decreases with size at least as $r^{-5}$ while the tidal force of the external wave grows as $r$, the end result is that the radiation reaction can become effectively negligible. This is the regime of Arnold diffusion in our problem and can lead to gravitational ionization as described in detail in our previous work [3, 4 .

It is possible that for the general $(m: 1)$ resonance other interesting phenomena may occur that are beyond the scope of this investigation.

\section{APPENDIX A: ABSORPTION OF GRAVITATIONAL WAVES}

Consider a plane monochromatic gravitational wave with wave vector $\mathbf{K}$ and frequency $\Omega=c|\mathbf{K}|$. The spacetime metric is given by $g_{\mu \nu}=\eta_{\mu \nu}+\epsilon h_{\mu \nu}$, where we impose the gauge condition $h_{0 \mu}=0$. Here $\eta_{\mu \nu}$ is the Minkowski metric with signature +2 ; moreover, it is important to note that our gauge has the characteristic property that the worldline of a static test particle is a geodesic and this is consistent with the binary system as a whole remaining at rest in this external radiation field. The gravitational wave amplitude is then characterized by a symmetric and traceless $h_{i j}$ with $\partial_{j} h_{i j}=0$ and $\square h_{i j}=0$. Let

$$
h_{i j}=\operatorname{Re}\left[\hat{h}_{i j} \exp (-i \Omega t+i \mathbf{K} \cdot \mathbf{x})\right],
$$

where $\hat{h}_{i j} K^{j}=0$. We are interested in the gravitational influence of this wave on a Keplerian binary system. If the wavelength of the radiation is much larger than the dimension of the system, then the interaction of the wave with the system is predominantly tidal in character. Then the tidal matrix is given by

$$
\mathcal{K}_{i j}=-\frac{1}{2} \frac{\partial^{2} h_{i j}}{\partial t^{2}}(t, \mathbf{0}),
$$

since the center of mass of the binary system is fixed at the origin of the spatial coordinates. We choose the coordinate system such that the wave is incident in the $(x, z)$-plane; hence, $\hat{\mathbf{K}}=(\sin \Theta, 0, \cos \Theta)$ is the unit propagation vector of the wave. Let us then choose $(\hat{\mathbf{K}}, \hat{\mathbf{N}}, \hat{\mathbf{y}})$ to be an orthonormal triad with $\hat{\mathbf{N}}=(\cos \Theta, 0,-\sin \Theta)$. Expanding 
the symmetric and traceless $\hat{h}_{i j}$ in terms of this basis triad and taking the transversality of the wave into account $\left(\hat{h}_{i j} \hat{K}^{j}=0\right)$, we find

$$
\hat{h}_{i j}=2 \alpha\left(\hat{N}_{i} \hat{N}_{j}-\hat{y}_{i} \hat{y}_{j}\right)+2 \beta \exp \left(-i \phi_{0}\right)\left(\hat{N}_{i} \hat{y}_{j}+\hat{N}_{j} \hat{y}_{i}\right),
$$

where $2 \alpha$ and $2 \beta$ are the real amplitudes of the two independent linear polarization states and $\phi_{0}$ is the constant phase difference between them. Here the zero of time is so chosen that $\alpha$ is real. It is now possible to recover equation (4) from (A1)- (A3).

The absorption of tidal gravitational energy from such a wave is not unidirectional in general; that is, a system can gain or lose energy as a result of its tidal interaction with an incident gravitational wave. On the other hand, we have found that during resonance capture the system always gains energy.

The nonlinear evolution of the binary system has been emphasized in our recent work when the external perturbation can, in effect, be considered to be a plane monochromatic gravitational wave [1. 4 . However, we have not considered an arbitrary incident wave packet or a stochastic background of gravitational waves. These cases have been treated in the absence of radiation damping for the linear evolution of the binary system [9]. It turns out that for a binary immersed in a random, isotropic and unpolarized linear gravitational wave background characterized solely by its energy spectral density, the osculating elements of the relative orbit undergo random walks [9]. The extension of these results to the long term nonlinear evolution of the system remains a task for the future. In this connection, let us mention that nonlinear stochastic differential equations have been the subject of numerous investigations (see, for example, [10,11]).

\section{APPENDIX B: EQUATIONS OF MOTION IN DELAUNAY'S VARIABLES}

The equations of motion in terms of Delaunay's action-angle variables are of basic importance for the dynamical considerations in this paper. The standard treatments usually involve Hamiltonian systems; therefore, we present here a direct derivation of these equations since our system is dissipative.

The equations of motion for the perturbed Kepler problem (11) can be written in vector form as

$$
\frac{d^{2} \mathbf{x}}{d t^{2}}+\frac{\mathbf{x}}{r^{3}}=\mathbf{F},
$$

where $r=|\mathbf{x}|$ and $\mathbf{F}$ is given by

$$
\begin{aligned}
F^{i}= & -\epsilon \mathcal{K}_{i j} x^{j}-\frac{\delta}{r^{3}}\left[\left(12 v^{2}-30 \dot{r}^{2}-\frac{4}{r}\right) v^{i}\right. \\
& \left.-\frac{\dot{r}}{r}\left(36 v^{2}-50 \dot{r}^{2}+\frac{4}{3 r}\right) x^{i}\right] .
\end{aligned}
$$

At any instant of time $t, \mathbf{x}$ and $\mathbf{v}$ specify the instantaneous orbital plane that contains the osculating ellipse with its focus always at the origin of coordinates. Let us choose the coordinate system such that the osculating ellipse at time $t$ has the form given in figure 1 .

To obtain the equations of motion in Delaunay elements, we employ an orthonormal frame field adapted to the instantaneous osculating ellipse, write the component equations of motion using this basis, and perform a transformation from the inertial Cartesian coordinates to those adapted to the instantaneous osculating ellipse. We define the frame field as follows. Let $\hat{\mathbf{r}}$ be a unit radial vector, $\mathbf{x}=r \hat{\mathbf{r}}$, and $\hat{\mathbf{n}}$ be a unit vector normal to the plane of the instantaneous osculating ellipse. To complete this frame field, we include $\hat{\mathbf{s}}=\hat{\mathbf{n}} \times \hat{\mathbf{r}}$ which is a unit vector in the orbital plane normal to $\hat{\mathbf{r}}$. Then $\mathbf{F}$ can be written as

$$
\mathbf{F}=F_{r} \hat{\mathbf{r}}+F_{s} \hat{\mathbf{s}}+F_{n} \hat{\mathbf{n}}
$$

using its radial, sideways and normal components. In what follows, we use the definition of the Delaunay elements $(L, G, H, \ell, g, h)$ given by

$$
\begin{aligned}
L & =a^{1 / 2}, \\
G & =L\left(1-e^{2}\right)^{1 / 2},
\end{aligned}
$$




$$
\begin{aligned}
H & =G \cos i \\
\ell & =\hat{u}-e \sin \hat{u} \\
g & =\operatorname{argument} \text { of the periastron, } \\
h & =\text { longitude of the ascending node, }
\end{aligned}
$$

where $a$ is the semimajor axis of the osculating ellipse, $e$ is its eccentricity, $\hat{u}$ is the eccentric anomaly, and $\ell$ is the mean anomaly. Only positive square roots are considered throughout this paper. The equation for the radial position $r$ in terms of the true anomaly $\hat{v}$ is given by

$$
r=\frac{a\left(1-e^{2}\right)}{1+e \cos \hat{v}}
$$

The orbital energy (11) is given by

$$
E=\frac{1}{2} v^{2}-\frac{1}{r}=-\frac{1}{2 a}
$$

where

$$
v^{2}=\dot{r}^{2}+\frac{a\left(1-e^{2}\right)}{r^{2}}
$$

using the fact that $G=|\mathbf{x} \times \mathbf{v}|$. It follows from these relations and (B5) that

$$
\dot{r}=\frac{e \sin \hat{v}}{G}
$$

It is clear from (B1) that

$$
\frac{d E}{d t}=\mathbf{F} \cdot \mathbf{v}
$$

It then follows from $(\overline{B 7}),(\mathrm{B} 8)$, and the definition of $L$ that we have

$$
\frac{d L}{d t}=L^{3} \mathbf{F} \cdot \mathbf{v}=\frac{a}{\left(1-e^{2}\right)^{1 / 2}}\left[F_{r} e \sin \hat{v}+F_{s} \frac{a\left(1-e^{2}\right)}{r}\right] .
$$

To obtain the equations of motion for the remaining Delaunay elements, we use the relations

$$
\mathbf{x} \times \mathbf{v}=G \hat{\mathbf{n}}, \quad \frac{d}{d t}(\mathbf{x} \times \mathbf{v})=\mathbf{x} \times \mathbf{F}
$$

to get

$$
\frac{d G}{d t}=r F_{s}
$$

and

$$
G \frac{d \hat{\mathbf{n}}}{d t}=-r F_{n} \hat{\mathbf{s}} .
$$

Now consider a coordinate system $\left(x^{\prime}, y^{\prime}, z^{\prime}\right)$ oriented to the instantaneous osculating ellipse such that the perturbed motion is given by $x^{\prime}=r \cos (\hat{v}+g), y^{\prime}=r \sin (\hat{v}+g)$, and $z^{\prime}=0$. The transformation from the coordinate system $(x, y, z)$ to $\left(x^{\prime}, y^{\prime}, z^{\prime}\right)$ consists of a rotation by an angle $h$ about the $z$-axis followed by a rotation by the inclination angle $i$ about the line of the ascending node, which is the $x^{\prime}$-axis. This transformation can be written as

$$
\left(\begin{array}{l}
x \\
y \\
z
\end{array}\right)=\left(\begin{array}{ccc}
\cos h & -\sin h \cos i & \sin h \sin i \\
\sin h & \cos h \cos i & -\cos h \sin i \\
0 & \sin i & \cos i
\end{array}\right)\left(\begin{array}{l}
x^{\prime} \\
y^{\prime} \\
z^{\prime}
\end{array}\right) .
$$

It follows that the relative position is given by 


$$
\begin{aligned}
x=r \sin \vartheta \cos \varphi & =r[\cos h \cos (\hat{v}+g)-\sin h \cos i \sin (\hat{v}+g)], \\
y=r \sin \vartheta \sin \varphi & =r[\sin h \cos (\hat{v}+g)+\cos h \cos i \sin (\hat{v}+g)], \\
z=r \cos \vartheta & =r \sin i \sin (\hat{v}+g) .
\end{aligned}
$$

In the $\left(x^{\prime}, y^{\prime}, z^{\prime}\right)$ coordinate system, $\hat{\mathbf{n}}=(0,0,1)$. Thus in the inertial frame,

$$
\hat{\mathbf{n}}=(\sin h \sin i,-\cos h \sin i, \cos i),
$$

and

$$
\begin{aligned}
\hat{\mathbf{s}}=\hat{\mathbf{n}} \times \hat{\mathbf{r}} & \\
= & (-\cos h \sin (\hat{v}+g)-\sin h \cos i \cos (\hat{v}+g), \\
& \quad \cos i \cos h \cos (\hat{v}+g)-\sin h \sin (\hat{v}+g), \sin i \cos (\hat{v}+g)) .
\end{aligned}
$$

Substituting (B14) into (B11) results in

$$
\begin{aligned}
\frac{d i}{d t} & =\frac{r F_{n}}{G} \cos (\hat{v}+g), \\
\frac{d h}{d t} \sin i & =\frac{r F_{n}}{G} \sin (\hat{v}+g) .
\end{aligned}
$$

From the definition of $H$ in $(\mathrm{B} 4)$ and equations $(\mathrm{B} 10)$ and $(\vec{B} 15)$, it follows that

$$
\frac{d H}{d t}=r\left[F_{s} \cos i-F_{n} \sin i \cos (\hat{v}+g)\right]
$$

The dynamical equation for the mean anomaly is obtained by exactly the same method as used in [1] (cf. appendix $\mathrm{B}$ in [1]). The result for the three dimensional case is obtained by replacing $F_{\theta}$ in [1] by $F_{s}$; hence,

$$
\frac{d \ell}{d t}=\omega+\frac{r}{e(a)^{1 / 2}}\left[F_{r}\left(-2 e+\cos \hat{v}+e \cos ^{2} \hat{v}\right)-F_{s}(2+e \cos \hat{v}) \sin \hat{v}\right] .
$$

To obtain the equation for the dynamics of the argument of the periastron, consider the relative velocity vector in the form

$$
\mathbf{v}=\dot{r} \hat{\mathbf{r}}+\frac{G}{r} \hat{\mathbf{s}}
$$

Project onto the $z$-axis to obtain

$$
\dot{z}=\dot{r} \sin i \sin (\hat{v}+g)+\frac{G}{r} \sin i \cos (\hat{v}+g) .
$$

Differentiating the $z$-component of $(\mathrm{B} 13)$ with respect to time and equating the result to (B18) gives

$$
\frac{d(\hat{v}+g)}{d t}=\frac{G}{r^{2}}-\frac{r F_{n}}{G} \frac{\cos i}{\sin i} \sin (\hat{v}+g) .
$$

Now, by taking the time derivative of the logarithm of (B5), we obtain

$$
\frac{d \hat{v}}{d t}=\frac{G}{r^{2}}-\frac{G}{e}\left[-F_{r} \cos \hat{v}+F_{s}\left(1+\frac{r}{a\left(1-e^{2}\right)}\right) \sin \hat{v}\right] .
$$

Subtracting (B20) from (B19) gives the dynamical equation for the argument of periastron as

$$
\begin{aligned}
\frac{d g}{d t}= & -\frac{r F_{n}}{G} \frac{\cos i}{\sin i} \sin (\hat{v}+g) \\
& +\frac{G}{e}\left[-F_{r} \cos \hat{v}+F_{s}\left(1+\frac{r}{a\left(1-e^{2}\right)}\right) \sin \hat{v}\right] .
\end{aligned}
$$

To summarize, the dynamical equations in Delaunay elements are 


$$
\begin{aligned}
\frac{d L}{d t}= & \frac{a}{\left(1-e^{2}\right)^{1 / 2}}\left[F_{r} e \sin \hat{v}+F_{s} \frac{a\left(1-e^{2}\right)}{r}\right], \\
\frac{d G}{d t}= & r F_{s}, \\
\frac{d H}{d t}= & r\left[F_{s} \cos i-F_{n} \sin i \cos (\hat{v}+g)\right], \\
\frac{d \ell}{d t}= & \omega+\frac{r}{e(a)^{1 / 2}}\left[F_{r}\left(-2 e+\cos \hat{v}+e \cos ^{2} \hat{v}\right)-F_{s}(2+e \cos \hat{v}) \sin \hat{v}\right], \\
\frac{d g}{d t}= & -\frac{r F_{n}}{G} \frac{\cos i}{\sin i} \sin (\hat{v}+g) \\
& +\frac{\left(a\left(1-e^{2}\right)\right)^{1 / 2}}{e}\left[-F_{r} \cos \hat{v}+F_{s}\left(1+\frac{r}{a\left(1-e^{2}\right)}\right) \sin \hat{v}\right], \\
\frac{d h}{d t}= & \frac{r F_{n} \frac{\sin (\hat{v}+g)}{G}}{\sin i} .
\end{aligned}
$$

It now remains to express $F_{r}, F_{s}$ and $F_{n}$ in terms of Delaunay elements.

The equations of motion in Delaunay's variables can be expressed in the form given in (14). To this end, let

$$
\mathcal{H}^{*}=\frac{1}{2} \mathcal{K}_{i j} x^{i} x^{j}
$$

be the Hamiltonian associated with the external perturbation and $\delta=\epsilon \Delta$. Then (B2) can be written as

$$
\mathbf{F}=-\epsilon \nabla \mathcal{H}^{*}+\epsilon \Delta \mathbf{f}
$$

where

$$
\mathbf{f}=\frac{\dot{r}}{r^{3}}\left(24 v^{2}-20 \dot{r}^{2}+\frac{16}{3 r}\right) \hat{\mathbf{r}}-\frac{G}{r^{4}}\left(12 v^{2}-30 \dot{r}^{2}-\frac{4}{r}\right) \hat{\mathbf{s}} .
$$

Thus $f_{r}=\dot{r}(\chi-\psi) / r^{3}, f_{s}=-G \psi / r^{4}$ and $f_{n}=0$. It is important to note that the functions $\psi$ and $\chi$ can be expressed solely in terms of Delaunay variables in the plane of the orbit $(L, G, \ell, g)$ just as in our previous work [1,22]. It then follows from the inspection of equations $(\overline{B 24})$ and $(\overline{B 25})$ that when radiation damping acts alone, the orbital plane remains fixed in space while the orbit tends to a circle as it shrinks $(e \rightarrow 0$ and $a \rightarrow 0)$. Using these results in (B22), one can show that equation (14) is recovered with appropriate definitions of the damping functions $f_{D}$ and the replacement of $\Omega t$ by $\tau$.

\section{APPENDIX C: PARTIAL AVERAGING}

This appendix is devoted to the determination of the resonant behavior of the three dimensional Kepler system when averaged over the binary period. Averaging over this "fast" motion should reveal the "slow" oscillatory behavior of the system while in resonance and its exit out of the resonance.

The perturbed Kepler system continuously emits gravitational waves and hence loses energy and angular momentum to gravitational radiation damping. Hence the semimajor axis of the osculating ellipse must steadily decrease except when the system is trapped in a resonance. At resonance, a delicate balance exists between the external gravitational perturbation and the radiation damping since the energy (and hence the semimajor axis) of the osculating ellipse would be constant on the average. That is, during resonance capture the energy deposited into the orbit by the external wave would equal on average the energy lost via the emission of gravitational radiation. In general, the system slowly drifts out of the resonance when this subtle balance is significantly upset.

Let $L=L_{0}$ when the resonance condition $m \omega=n \Omega$ is exactly satisfied; then, we assume

$$
\begin{aligned}
L & =L_{0}+\epsilon^{1 / 2} \mathcal{D}, \\
\ell & =\frac{1}{L_{0}^{3}} t+\phi,
\end{aligned}
$$


during resonance capture. As before [2], we recast the equations of motion in Delaunay variables (14) in terms of the new variables $(\mathcal{D}, \phi)$. To this end,

$$
\frac{1}{L^{3}}=\frac{1}{L_{0}^{3}}\left[1-3 \frac{\mathcal{D}}{L_{0}} \epsilon^{1 / 2}+6 \frac{\mathcal{D}^{2}}{L_{0}^{2}} \epsilon+\mathcal{O}\left(\epsilon^{3 / 2}\right)\right],
$$

and so we obtain from (14) upon Taylor expansion

$$
\begin{aligned}
\dot{\mathcal{D}} & =-\epsilon^{1 / 2} F_{11}-\epsilon \mathcal{D} F_{12} \\
\dot{G} & =-\epsilon F_{22} \\
\dot{H} & =-\epsilon F_{32} \\
\dot{\phi} & =-\epsilon^{1 / 2} \frac{3 \mathcal{D}}{L_{0}^{4}}+\epsilon\left(\frac{6 \mathcal{D}^{2}}{L_{0}^{5}}+F_{42}\right) \\
\dot{g} & =\epsilon F_{52} \\
\dot{h} & =\epsilon F_{62}
\end{aligned}
$$

where terms of order higher than $\epsilon$ have been neglected. Here $\left(F_{i j}\right)$ are defined by

$$
\begin{aligned}
& F_{11}=\frac{\partial \mathcal{H}^{*}}{\partial \ell}-\Delta f_{L}, \\
& F_{12}=\frac{\partial^{2} \mathcal{H}^{*}}{\partial L \partial \ell}-\Delta \frac{\partial f_{L}}{\partial L}, \\
& F_{22}=\frac{\partial \mathcal{H}^{*}}{\partial g}-\Delta f_{G}, \\
& F_{32}=\frac{\partial \mathcal{H}^{*}}{\partial h}-\Delta f_{H},
\end{aligned}
$$

while

$$
\begin{aligned}
& F_{42}=\frac{\partial \mathcal{H}^{*}}{\partial L}+\Delta f_{\ell}, \\
& F_{52}=\frac{\partial \mathcal{H}^{*}}{\partial G}+\Delta f_{g}, \\
& F_{62}=\frac{\partial \mathcal{H}^{*}}{\partial H}+\Delta f_{h} .
\end{aligned}
$$

Note that the first index in $F_{i j}$ refers to the equation in which it appears while the second index refers to its order in powers of the perturbation parameter $\epsilon^{1 / 2}$. Moreover, all the terms on the right sides of (C5) and (C6) are to be evaluated at $\left(L_{0}, G, H, \phi+n \Omega t / m, g, h\right)$ so that $\left(F_{i j}\right)$ become functions of $(G, H, \phi+n \Omega t / m, g, h, t)$, since $\mathcal{H}^{*}$ is explicitly dependent upon time. Let us further note that at resonance the appropriate perturbation parameter turns out to be $\epsilon^{1 / 2}$, since only with this choice would the conjugate variables $\mathcal{D}$ and $\phi$ both vary predominantly on the same slow temporal scale given by $\epsilon^{1 / 2} t$.

The system (C4) is periodic in $t$ with period $2 \pi m / \Omega$. To average this system over its period, we introduce an averaging transformation that has the function of rendering the transformed system (C4) in a form that is averaged to first order in $\epsilon^{1 / 2}$. The resulting system will then be replaced by the second order averaged system, where the terms of order $\epsilon$ are simply averaged over the $2 \pi \mathrm{m} / \Omega$ period. The averaging theorem ensures that the solution of the second order averaged system is sufficiently close to the solution of (C4) over a timescale of order $\epsilon^{-1 / 2}$ [2].

Let us first define the average of $F_{i j}$ to be

$$
\left\langle F_{i j}\right\rangle=\frac{\Omega}{2 \pi m} \int_{0}^{2 \pi m / \Omega} F_{i j}\left(G, H, \frac{n \Omega}{m} t+\phi, g, h, t\right) d t .
$$

Since the averaging transformation involves only first order quantities, the only term of interest in (C4) would then be $F_{11}$ and we let $\lambda(G, H, \phi, g, h, t)=F_{11}-\left\langle F_{11}\right\rangle$ be its deviation from the average. Next, we define $\Lambda(G, H, \phi, g, h, t)$ to be the antiderivative of $\lambda$ with respect to $t$ such that $\langle\Lambda\rangle=0$. The averaging transformation is then given by

$$
\mathcal{D}=\hat{\mathcal{D}}-\epsilon^{1 / 2} \Lambda(\hat{G}, \hat{H}, \hat{\phi}, \hat{g}, \hat{h}, t),
$$


$G=\hat{G}, H=\hat{H}, \phi=\hat{\phi}, g=\hat{g}$ and $h=\hat{h}$. Hence the averaging transformation is on average equivalent to the identity transformation. Upon this transformation, ( $\overline{\mathrm{C} 4}$ ) takes the form

$$
\begin{aligned}
\dot{\hat{\mathcal{D}}} & =-\epsilon^{1 / 2}\left\langle F_{11}\right\rangle-\epsilon \hat{\mathcal{D}}\left(\frac{3}{L_{0}^{4}} \frac{\partial \Lambda}{\partial \hat{\phi}}+F_{12}\right), \\
\dot{\hat{G}} & =-\epsilon F_{22}, \\
\dot{\hat{H}} & =-\epsilon F_{32}, \\
\dot{\hat{\phi}} & =-\epsilon^{1 / 2}\left(\frac{3}{L_{0}^{4}} \hat{\mathcal{D}}\right)+\epsilon\left(\frac{6}{L_{0}^{5}} \hat{\mathcal{D}}^{2}+\frac{3}{L_{0}^{4}} \Lambda+F_{42}\right), \\
\dot{\hat{g}} & =\epsilon F_{52}, \\
\dot{\hat{h}} & =\epsilon F_{62},
\end{aligned}
$$

where terms of order $\epsilon^{3 / 2}$ and higher have been neglected. We note that (Iי) is in averaged form to first order, but not to second order. Therefore, we now average (C9) to obtain the second order partially averaged system that we wish to study. To simplify the notation, we write our main result in the form

$$
\begin{aligned}
\dot{\mathcal{D}} & =-\epsilon^{1 / 2}\left\langle F_{11}\right\rangle-\epsilon \mathcal{D}\left\langle F_{12}\right\rangle, \\
\dot{G} & =-\epsilon\left\langle F_{22}\right\rangle, \\
\dot{H} & =-\epsilon\left\langle F_{32}\right\rangle, \\
\dot{\phi} & =-\epsilon^{1 / 2}\left(\frac{3}{L_{0}^{4}} \mathcal{D}\right)+\epsilon\left(\frac{6}{L_{0}^{5}} \mathcal{D}^{2}+\left\langle F_{42}\right\rangle\right), \\
\dot{g} & =\epsilon\left\langle F_{52}\right\rangle, \\
\dot{h} & =\epsilon\left\langle F_{62}\right\rangle,
\end{aligned}
$$

since $\langle\Lambda\rangle=0$ and $\langle\partial \Lambda / \partial \phi\rangle=0$. We remark that (C10) is simply the averaged form of our original system (C4); however, this will not be true in general but happens to be the case here. It now remains to compute the averages $\left\langle F_{i j}\right\rangle$ defined by (C7).

Let us first focus attention on the radiation reaction terms. It follows from the discussion in the last paragraph of appendix $\mathrm{B}$ that the effect of this frictional force is to maintain the orbital plane while the relative orbit loses energy and shrinks $(a \rightarrow 0, e \rightarrow 0)$. Therefore, the result of averaging is essentially the same as in our previous work [1].2] and is given by

$$
\begin{aligned}
\left\langle f_{L}\right\rangle & =-\frac{1}{G^{7}}\left(8+\frac{73}{3} e^{2}+\frac{37}{12} e^{4}\right), \\
\left\langle f_{G}\right\rangle & =-\frac{1}{L^{3} G^{4}}\left(8+7 e^{2}\right), \\
\left\langle f_{H}\right\rangle & =\left\langle f_{G}\right\rangle \cos i
\end{aligned}
$$

and $\left\langle f_{\ell}\right\rangle=\left\langle f_{g}\right\rangle=\left\langle f_{h}\right\rangle=0$. Moreover, $\left\langle\partial f_{L} / \partial L\right\rangle=\partial\left\langle f_{L}\right\rangle / \partial L$ and hence

$$
\left\langle\frac{\partial f_{L}}{\partial L}\right\rangle=-\frac{1}{3 L^{3} G^{5}}\left(146+37 e^{2}\right) .
$$

All these quantities are to be evaluated at $L=L_{0}$.

We consider next the terms involving the external Hamiltonian $\epsilon \mathcal{H}^{*}$, which must first be expressed in terms of the Delaunay elements. To this end, let

$$
\begin{aligned}
S(h) & =\sin h, \\
U(G, H, h) & =\cos \Theta \cos i S+\sin \Theta \sin i, \\
V(G, H, h) & =\cos h \cos i, \\
W(h) & =\cos \Theta \cos h,
\end{aligned}
$$

and define $P_{\sigma}$ and $\tilde{P}_{\sigma}, \sigma=0, \pm$, such that 


$$
\begin{aligned}
P_{0} & =\frac{1}{2}\left(-S^{2}+U^{2}-V^{2}+W^{2}\right), \\
P_{+} & =\frac{1}{2}\left(-S^{2}-U^{2}+V^{2}+W^{2}\right), \\
P_{-} & =-S V-U W, \\
\tilde{P}_{0} & =S W-U V, \\
\tilde{P}_{+} & =S W+U V, \\
\tilde{P}_{-} & =-S U+V W .
\end{aligned}
$$

Using these quantities, we can form $\mathcal{P}_{\sigma}(G, H, h, t)$,

$$
\mathcal{P}_{\sigma}=\frac{1}{2} \alpha \Omega^{2} \cos \Omega t P_{\sigma}+\frac{1}{2} \beta \Omega^{2} \cos \left(\Omega t+\phi_{0}\right) \tilde{P}_{\sigma} .
$$

It is now possible to express $\mathcal{H}^{*}$ in terms of the Delaunay variables as follows:

$$
\mathcal{H}^{*}=\sum_{\sigma=0, \pm} \mathcal{P}_{\sigma} \mathcal{Q}^{\sigma}
$$

where

$$
\begin{aligned}
& \mathcal{Q}^{0}(L, G, \ell, g)=r^{2}=a^{2}\left(1+\frac{3}{2} e^{2}\right)-4 a^{2} \sum_{\nu=1}^{\infty} \frac{1}{\nu^{2}} J_{\nu}(\nu e) \cos \nu \ell \\
& \mathcal{Q}^{+}(L, G, \ell, g)=r^{2} \cos (2 \hat{v}+2 g)=\frac{5}{2} a^{2} e^{2} \cos 2 g+a^{2} \sum_{\nu=1}^{\infty}\left(A_{\nu} \cos 2 g \cos \nu \ell-B_{\nu} \sin 2 g \sin \nu \ell\right), \\
& \mathcal{Q}^{-}(L, G, \ell, g)=r^{2} \sin (2 \hat{v}+2 g)=\frac{5}{2} a^{2} e^{2} \sin 2 g+a^{2} \sum_{\nu=1}^{\infty}\left(A_{\nu} \sin 2 g \cos \nu \ell+B_{\nu} \cos 2 g \sin \nu \ell\right) .
\end{aligned}
$$

Here $A_{\nu}$ and $B_{\nu}$ are functions of the eccentricity $e=\left(L^{2}-G^{2}\right)^{1 / 2} / L$ and can be expressed in terms of the Bessel function $J_{\nu}(x)$ as

$$
\begin{aligned}
& A_{\nu}=\frac{4}{\nu^{2} e^{2}}\left[2 \nu e\left(1-e^{2}\right) J_{\nu}^{\prime}(\nu e)-\left(2-e^{2}\right) J_{\nu}(\nu e)\right], \\
& B_{\nu}=-\frac{8}{\nu^{2} e^{2}}\left(1-e^{2}\right)^{1 / 2}\left[e J_{\nu}^{\prime}(\nu e)-\nu\left(1-e^{2}\right) J_{\nu}(\nu e)\right],
\end{aligned}
$$

where $J_{\nu}^{\prime}(x)=d J_{\nu}(x) / d x$.

We are interested in the average value of $\mathcal{H}^{*}$; therefore, it is convenient to express $\mathcal{P}_{\sigma}$ as

$$
\mathcal{P}_{\sigma}=\mathcal{C}_{\sigma} \cos \Omega t+\mathcal{S}_{\sigma} \sin \Omega t
$$

where $\mathcal{C}_{\sigma}$ and $\mathcal{S}_{\sigma}$ can be explicitly determined using (C17). Moreover, it proves convenient to express the Fourier series expansions of $\mathcal{Q}^{\sigma}$ collectively as

$$
\mathcal{Q}^{\sigma}=a_{0}^{\sigma}+\sum_{\nu=1}^{\infty}\left(a_{\nu}^{\sigma} \cos \nu \ell+b_{\nu}^{\sigma} \sin \nu \ell\right) .
$$

The average of $\mathcal{H}^{*}$,

$$
\left\langle\mathcal{H}^{*}\right\rangle=\frac{\Omega}{2 \pi m} \int_{0}^{2 \pi m / \Omega} \mathcal{H}^{*}\left(L, G, H, \frac{n \Omega}{m} t+\phi, g, h, t\right) d t
$$

is given by

$$
\left\langle\mathcal{H}^{*}\right\rangle=\mathcal{T}_{c}(L, G, H, g, h) \cos m \phi+\mathcal{T}_{s}(L, G, H, g, h) \sin m \phi
$$


where

$$
\begin{aligned}
& \mathcal{T}_{c}=\frac{1}{2} \sum_{\sigma}\left(a_{m}^{\sigma} \mathcal{C}_{\sigma}+b_{m}^{\sigma} \mathcal{S}_{\sigma}\right), \\
& \mathcal{T}_{s}=\frac{1}{2} \sum_{\sigma}\left(-a_{m}^{\sigma} \mathcal{S}_{\sigma}+b_{m}^{\sigma} \mathcal{C}_{\sigma}\right),
\end{aligned}
$$

for $n=1$. If $n \neq 1$, then $\left\langle\mathcal{H}^{*}\right\rangle=0$; hence, we define $\mathcal{T}_{c}=\mathcal{T}_{s}=0$ in this case.

It is now possible to express the quantities in (C10) that depend on the external perturbation in terms of $\mathcal{T}_{c}$ and $\mathcal{T}_{s}$. For instance, it is straightforward to use the formulas (C18), (C23) and (C25) to prove that

$$
\left\langle\frac{\partial \mathcal{H}^{*}}{\partial \ell}\right\rangle=\frac{\partial}{\partial \phi}\left\langle\mathcal{H}^{*}\right\rangle \text {. }
$$

Moreover, the other partial derivatives of $\mathcal{H}^{*}$ with respect to the other Delaunay variables simply commute with the operation of averaging. For instance, $\left\langle\partial \mathcal{H}^{*} / \partial L\right\rangle=\partial\left\langle\mathcal{H}^{*}\right\rangle / \partial L$, which is finally evaluated at $L=L_{0}$. The second order partially averaged equations would thus have a form similar to that for the planar case except for additional equations for the slow variation of the extra Delaunay variables $H$ and $h$. More explicitly, the partially averaged equations for the three dimensional Kepler system are given by equations (19) of [2] (with $L_{*} \rightarrow L_{0}, \varphi \rightarrow \phi, T_{c} \rightarrow \mathcal{T}_{c}$ and $T_{s} \rightarrow \mathcal{T}_{s}$ ) together with

$$
\begin{aligned}
\dot{H} & =-\epsilon\left[\frac{\partial \mathcal{T}_{c}}{\partial h} \cos m \phi+\frac{\partial \mathcal{T}_{s}}{\partial h} \sin m \phi+\frac{\Delta}{L_{0}^{3} G^{4}}\left(8+7 e^{2}\right) \cos i\right], \\
\dot{h} & =\epsilon\left(\frac{\partial \mathcal{T}_{c}}{\partial H} \cos m \phi+\frac{\partial \mathcal{T}_{s}}{\partial H} \sin m \phi\right) .
\end{aligned}
$$

The full set of partially averaged Delaunay equations is given by (15).

[1] Chicone C, Mashhoon B and Retzloff D G 1997 Gravitational ionization: a chaotic net in the Kepler system Class. Quantum Grav. 14 699-723

[2] Chicone C, Mashhoon B and Retzloff D G 1997 Evolutionary dynamics while trapped in resonance: a Keplerian binary system perturbed by gravitational radiation Class. Quantum Grav. 14 1831-1850

[3] Chicone C, Mashhoon B and Retzloff D G 1996 Gravitational ionization: periodic orbits of binary systems perturbed by gravitational radiation Ann. Inst. H. Poincaré, Phys. Théor. 64 87-125

[4] Chicone C, Mashhoon B and Retzloff D G 1996 On the ionization of a Keplerian binary system by periodic gravitational radiation J. Math. Phys. 37 3997-4016; 1997 Addendum ibid. 38544

[5] Hulse R A and Taylor J H 1975 Discovery of a pulsar in a binary system Astrophys. J. 195 L51-L53

[6] Taylor J H, Wolszczan A, Damour T and Weisberg J M 1992 Experimental constraints on strong-field relativistic gravity Nature 355 132-136

[7] Kopeikin S M 1994 Supplementary parameters in the parametrized post-Keplerian formalism Astrophys. J. 434 L67-L70 Doroshenko O V and Kopeikin S M 1995 Relativistic effect of gravitational deflection of light in binary pulsars Mon. Not. R. Astron. Soc. 274 1029-1038

Kopeikin S M 1996 Proper motion of binary pulsars as a source of secular variations of orbital parameters Astrophys. J. 467 L93-L95

Wex N and Kopeikin S M 1998 Frame dragging and other precessional effects in the stellar mass black hole-pulsar binaries preprint

[8] Arzoumanian Z, Phillips J A, Taylor J H and Wolszczan A 1996 Radio beam of the relativistic binary pulsar B1534+12 Astrophys. J. 470 1111-1117

Wolszczan A 1997 Relativistic binary pulsars Relativistic Gravitation and Gravitational Radiation ed J-A Marck and J-P Lasota (Cambridge: Cambridge University Press) pp 113-128

[9] Mashhoon B 1978 On tidal resonance Astrophys. J. 223 285-298

Mashhoon B 1977 Tidal radiation Astrophys. J. 216 591-609

Mashhoon B 1979 On the detection of gravitational radiation by the Doppler tracking of spacecraft Astrophys. J. 227 1019-1036 
Mashhoon B 1980 Absorption of gravitational radiation GRG Abstracts (Jena meeting) pp 1505-1507

Mashhoon B, Carr B J and Hu B L 1981 The influence of cosmological gravitational waves on a Newtonian binary system Astrophys. J. 246 569-591

[10] Arnold L 1974 Stochastic Differential Equations: Theory and Applications (New York: Wiley-Interscience)

[11] Friedman A 1975 Stochastic Differential Equations and Applications (Vol 1-2) (New York: Academic Press)

FIG. 1. Schematic plot of the osculating ellipse in the three dimensional Kepler problem. The instantaneous position vector is $\mathbf{x}(t)=(x, y, z)$. The unit vector $\hat{\mathbf{n}}$ is normal to the instantaneous orbital plane and points in the same direction as the orbital angular momentum.

FIG. 2. The plots are for system (7) with parameter values $\epsilon=10^{-4}, \delta / \epsilon=10^{-3}, \alpha=2, \beta=2, \phi_{0}=0$ and $\Theta=\pi / 10$. Here, $L_{0}=(4 / 3)^{1 / 2} \approx 1.1547$ corresponds to $(1: 1)$ resonance with $\Omega=1 / L_{0}^{3}$. The top panel shows $L=a^{1 / 2}$ versus time $t$ for the initial conditions $\left(p_{\rho}, p_{\theta}, p_{z}, \rho, \theta, z\right)$ equal to $(0.17554040552969,0.57754077894430,-0.050059905721582,2.7059982365422$, $-6.7506347480153,0.14058165333520)$. The middle panel shows cos $i$ versus time and the bottom panel shows $e$ versus $t$.

FIG. 3. The plots are for system (7) with the initial values of $\left(p_{\rho}, p_{\theta}, p_{z}, \rho, \theta, z\right)$ equal to $(-0.6579651108853,0.74664767379829,-0.12269473267506,1.4254331835434,-10.1974793053511,-0.052493593111567)$ and parameter values $\epsilon=10^{-3}, \delta / \epsilon=10^{-5}, \alpha=2, \beta=2, \phi_{0}=0, \Theta=\pi / 6$, and $\Omega=2 / L_{0}^{3}$ with $L_{0}=(4 / 3)^{1 / 2}$ so that the initial value is near the $(2: 1)$ resonance. The top panel depicts $L$ versus time, the middle panel depicts $G$ versus time, and the bottom panel depicts the eccentricity $e$ versus time. Every 10 th iterate of the $2 \pi / \Omega$ stroboscopic Poincaré map is plotted.

FIG. 4. The top panel depicts cos $i$ versus time, the middle panel depicts $G$ versus $L$ and the bottom panel depicts $H$ versus $L$ with the same data as in figure 3 .

FIG. 5. The plots are for system (7) with parameters as in figure 3 but with initial values of $\left(p_{\rho}, p_{\theta}, p_{z}, \rho, \theta, z\right)$ equal to $(-0.013456419026,0.510820155326,0.161909540107,2.35369782619,-644207.714939,-2.17211570796)$. The top panel depicts $L$ versus time, the middle panel depicts $G$ versus time, and the bottom panel depicts the eccentricity $e$ versus time. Every 10 th iterate of the $2 \pi / \Omega$ stroboscopic Poincaré map is plotted.

FIG. 6. The top panel depicts $\cos i$ versus time, the middle panel depicts $G$ versus $L$ and the bottom panel depicts $H$ versus $L$ with the same data as in figure 5. 


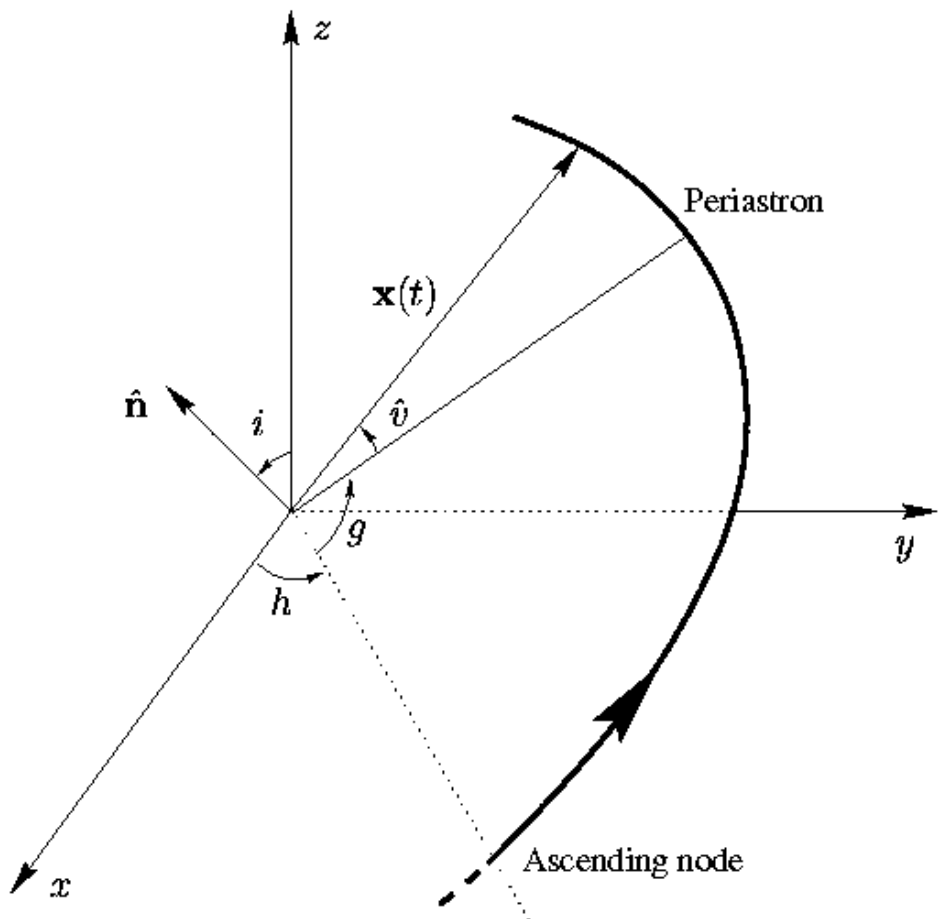

FIG. 1. 

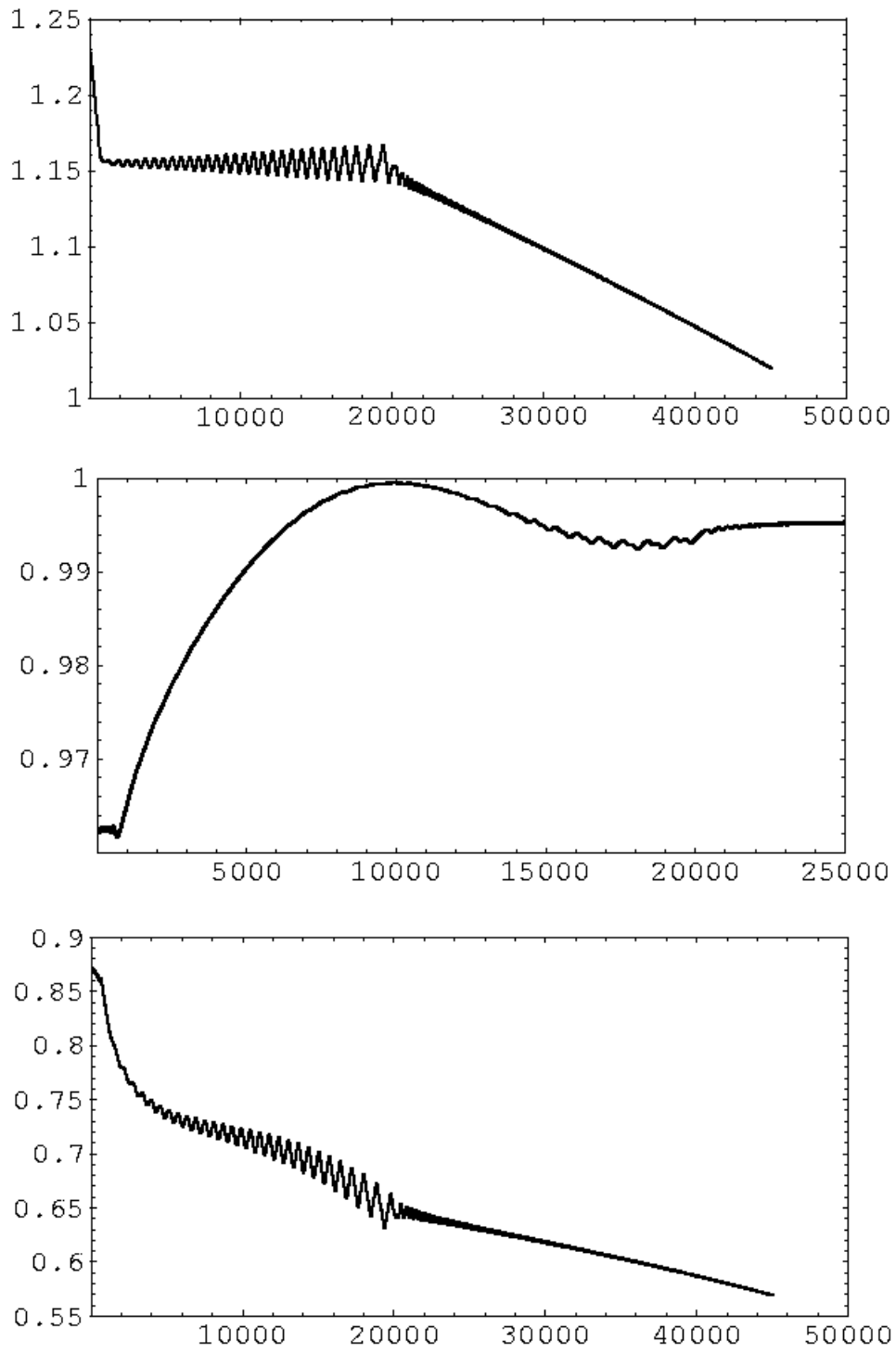

FIG. 2. 

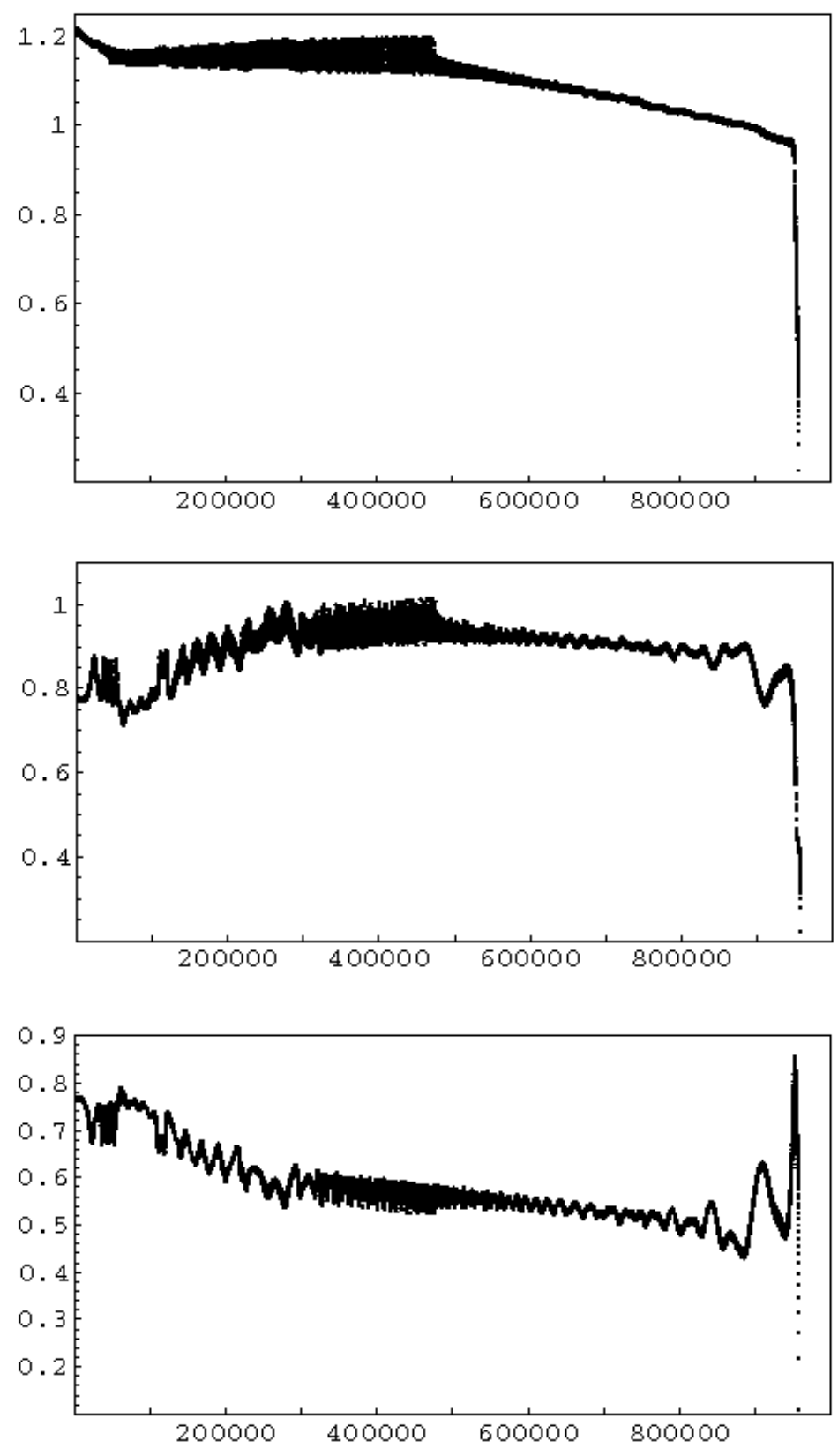

FIG. 3.

19 

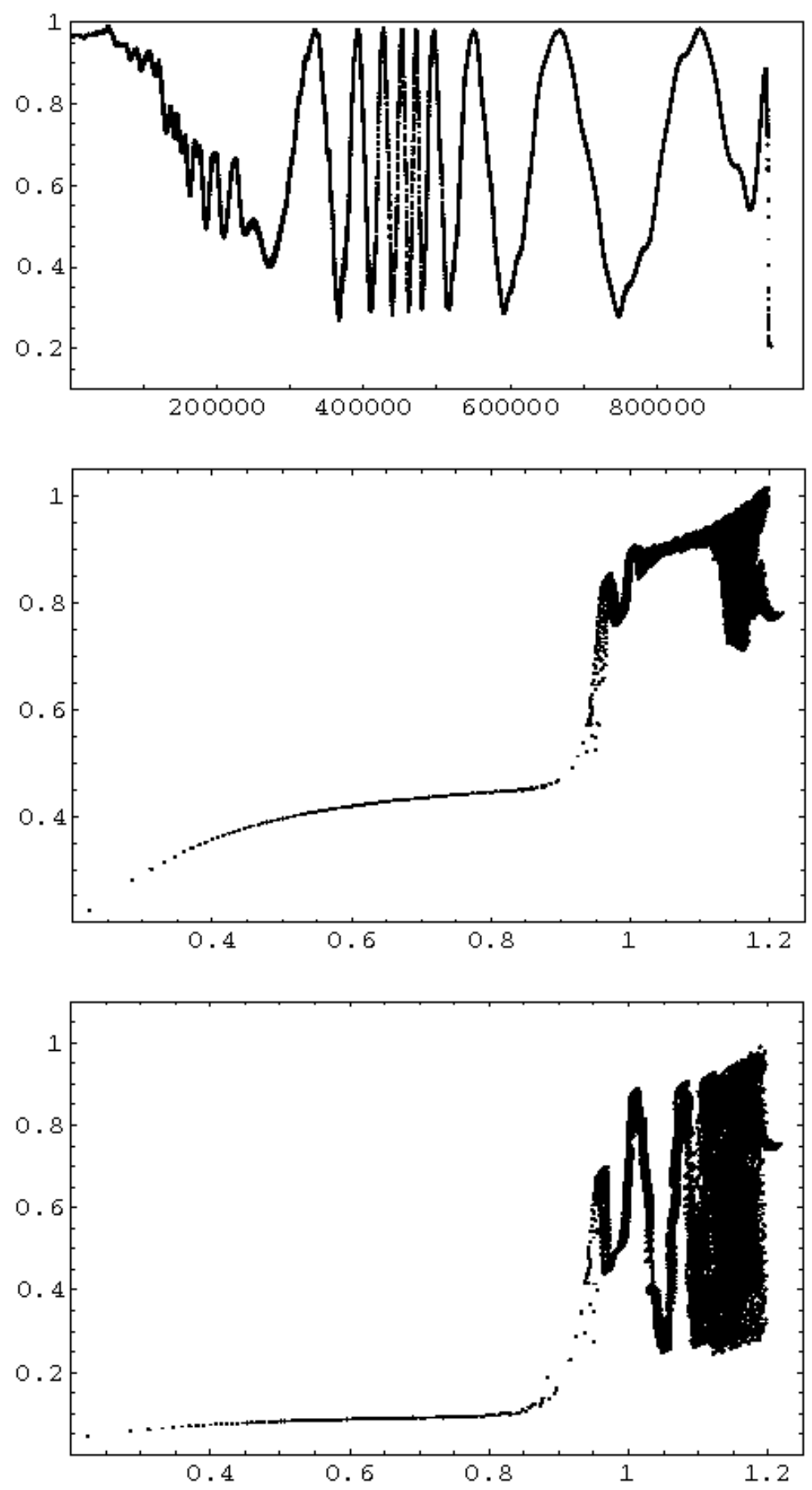

FIG. 4. 


$$
=
$$



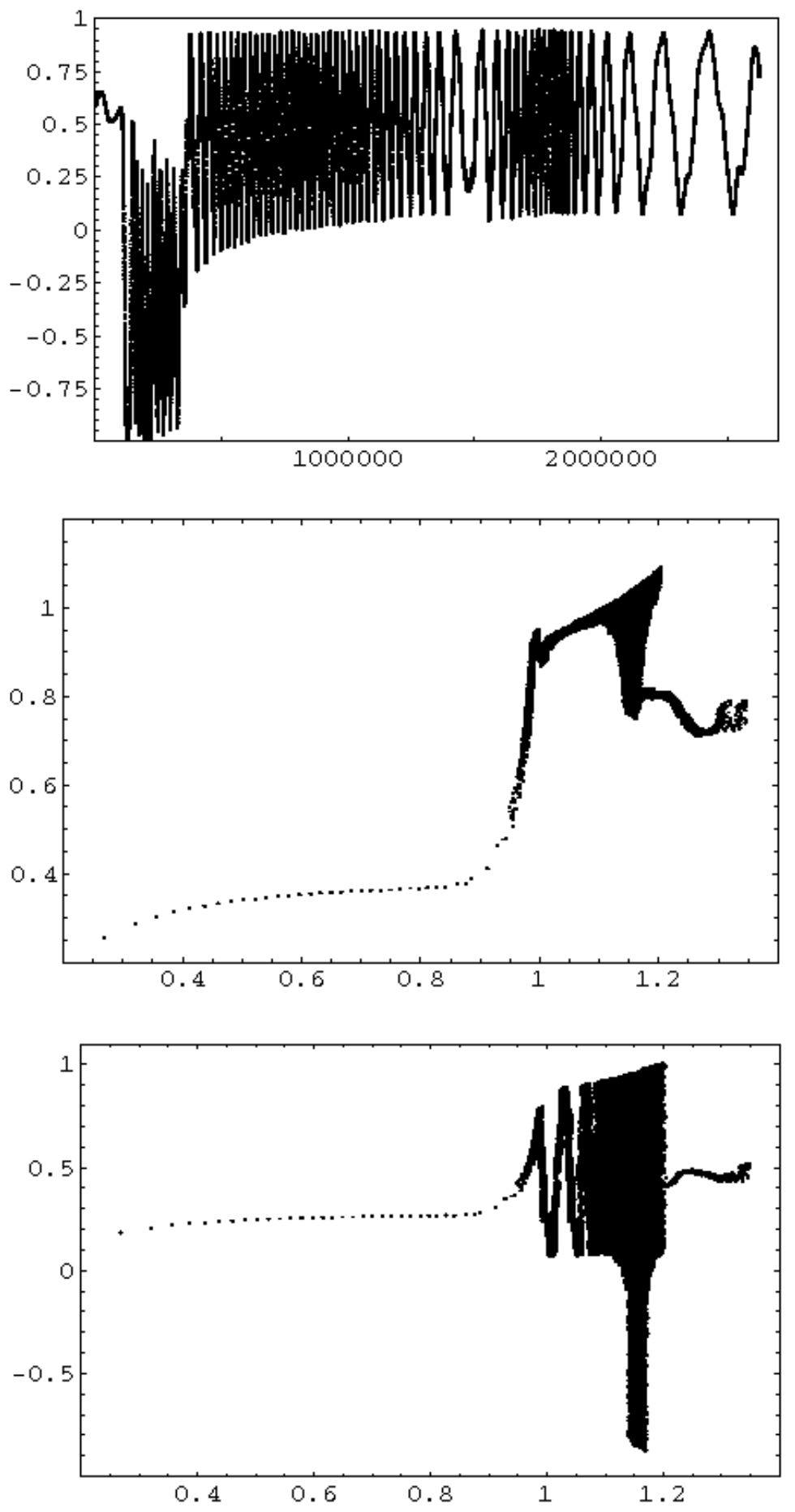

FIG. 6. 